\title{
Material encounters and media events: what kind of mathematics can a body do?
}

\author{
Elizabeth de Freitas ${ }^{1}$
}

Published online: 28 November 2015

(C) The Author(s) 2015. This article is published with open access at Springerlink.com

\begin{abstract}
This paper contributes to research on the material dimensions of teaching and learning mathematics, arguing that perception is not sensory integration or synthesis of multi-modal information, but rather a speculative investment in specific material encounters. This approach entails sociopolitical consequences for how we work with dis/ability in mathematics classrooms because it foregrounds how the relationship between sense (as meaning) and sense (as sensation) can be fundamentally altered. I focus on how visual perception in mathematics can be reconceived in these terms, drawing principally on the philosophical insights of Michel Serres and Brian Massumi. This paper shows how philosophical work on perception can shed light on embodied mathematics, opening up discussion about the potentiality of the human body.
\end{abstract}

Keywords Body $\cdot$ Perception $\cdot$ Sensation $\cdot$ Visualization $\cdot$ Diagrams $\cdot$ Philosophy $\cdot$ Materialism

\section{The unspecialized eye}

The mathematician Benoit Mandelbrot infamously declared "to see is to believe" when discussing his extensive use of computer imaging while inventing a new fractal geometry (Mandelbrot, 1982). Two years later, he was awarded the Barnard Medal for Meritorious Service to Science, where the judges celebrated this method: "In the great tradition of philosophers past, you looked at the world around you on a broader canvas." (Samuel, 2012, p. 21) Although Mandelbrot himself was not a programmer, the new fractal geometry emerged in consort with new computational technologies - as well as less impressive but pivotal enabling technologies, like the dot-matrix printer. These technologies, according to Mandelbrot, brought the eye back into mathematics:

Elizabeth de Freitas

1.de-freitas@mmu.ac.uk

1 Education and Social Research Institute, Manchester Metropolitan University, Manchester, UK 
The use of computer graphics is now in the process of altogether changing the role of the eye. The hard theoretical sciences had banished the eye for a long time, and many observers used to believe, and even hope, that it would remain banished forever. But computer graphics is bringing it back as an integral part of the very process of thinking, search and discovery. (Mandelbrot, 1992, p. 4)

The eye, according to Mandelbrot, was crucial to mathematical invention because of its potentiality or indeterminacy: "the eye is not specialized, it is a universal tool." (Samuel, 2012, p. 28) In other words, the eye can become differently abled, mutating into new habits of perception, in response to new media. The eye can be reconfigured as a new kind of sense organ, open to new ways of seeing and making sense - in other words, visual perception is "unspecialized" and experimental. Just as Mandelbrot came to see islands and landscapes in the speckled printer outputs, his colleague, the French mathematician Adrien Douady, momentarily dazed by the pages and pages of "computer-generated clouds of dots," came to see fractal mating rabbits (see Fig. 1). One can see in these instances of mathematical invention how visual perception is creative, experimental, and responsive. Drawing the rabbits onto the dot-matrix print-outs furnished new links between ideas and literally brought forth a new assemblage of meaning and matter. As Samuel (2012) notes, Douady emphasized the act of drawing in this process: "And just at that moment, quite suddenly, as soon as we had made the drawing, we understood what was going on." (Douady, 2005, quoted in Samuel, 2012, p. 45) Another scientist working on the same topic in the 1970s - Otto E. Rössler - argued that the material act of drawing was part of a feedback loop "directly influencing the properties of the mathematical objects under study.” (Samuel, 2012, p. 49) Rössler is known for his work on the

Fig. 1 Douady mating rabbits

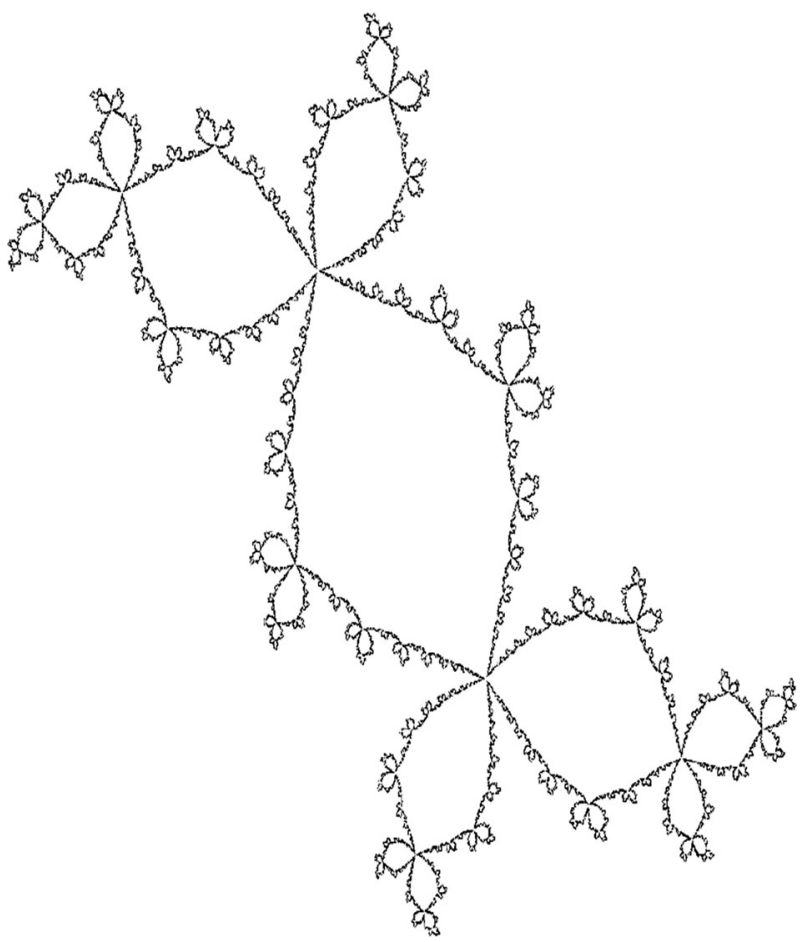


"Rössler attractor" in 1975, which was an emergent geometric shape representing long-term predictions within a chaotic system (Rössler, 1977). Part of his method of investigating chaotic systems involved drawing two spiral-like tracks on two sheets of paper and then manipulating the two sheets of paper in 3-space so that he might grasp how they were related (Fig. 2). He then created a series of sketches that stretched and distorted the spirals so that one can see how they moved across the two paper surfaces that were also bent and distorted.

Rössler states that the process of handling the paper was crucial in his thinking but that other bodily engagements were also entailed, including an audible ear "pop" and a "nose perception" by which he felt a string winding and winding around his nose as he worked the spiral on the page (Samuel, 2012). One might be inclined to simply dismiss such a statement as merely metaphoric, as though the reference to a body experience (string, nose, ear pop) stood in for the disembodied act of cognition, as though he was using a rhetorical device to help communicate the feeling of engagement. After all, there was no real string! And yet the encounter with paper and pen, the manual activity of folding and twisting, combined with the visual activity of glancing and squinting, entailed material actions that actually brought forth the new mathematical concepts. Rössler seems to be describing how sense (as meaning) and sense (as sensation) are melded together, emphasizing the power of material engagements with media. In the quote below, he draws attention to the haptic nature of vision, how looking is

Fig. 2 Rössler Attractors

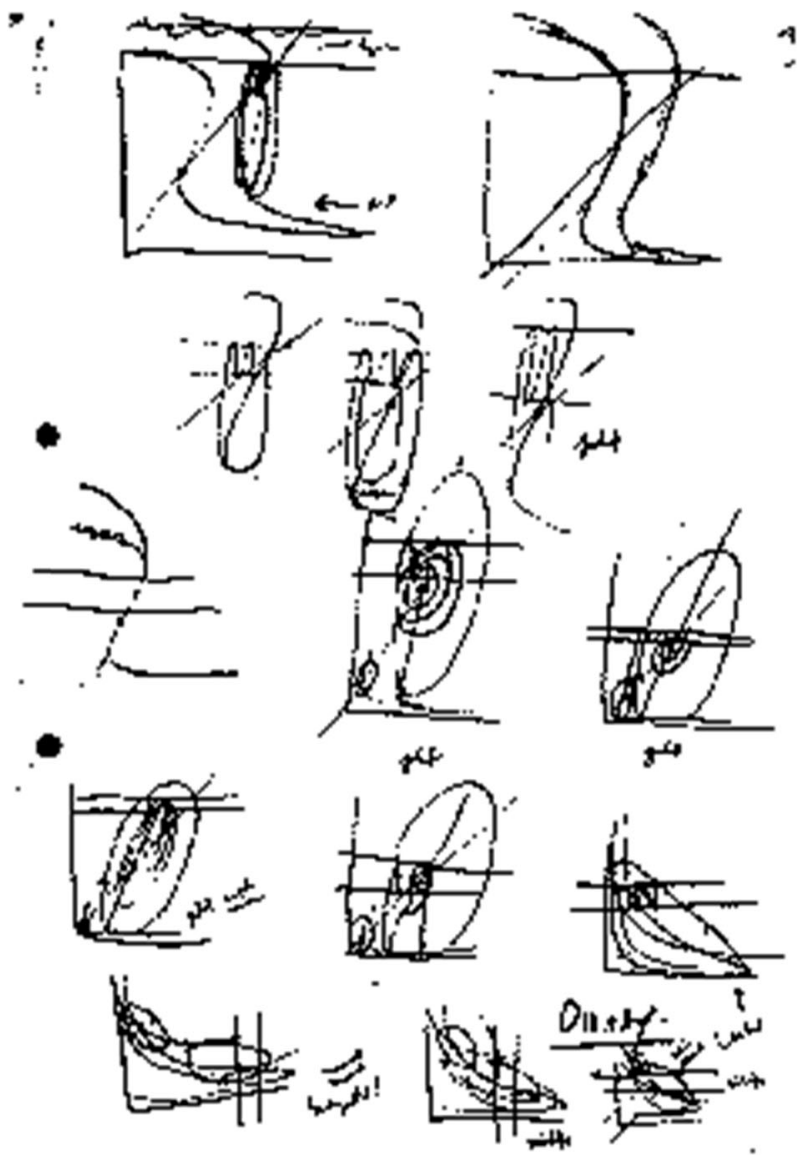


"completely tactile" and how the specificity of the medium makes a difference-"materiality does matter":

Real pictures are always erotic pictures as well. Topology or geometry is like looking from all sides as though in outer space. That is all completely tactile. Without touching and without kneading it is impossible to imagine. Materiality does matter: Essentially mathematics is nothing more than pottery. (Rössler, 2012/2007)

In these accounts of mathematical invention, we see an emphasis on the material encounters entailed in diagramming and doing mathematics. We also learn that mathematics is a highly embodied engagement with various kinds of media, and that new mathematical concepts emerge when the senses operate in unexpected ways. In the development of fractal geometry, Mandelbrot's unspecialized eye became reassembled with the drawing hand and the new digital media in ways that broke with current regimes of sense-making. The case of fractal geometry highlights how these mathematicians were part of a historical reconfiguring of the ontology of the senses, quite literally partaking in a cultural development or change whereby new capacities for the body were unfolding as the eye-hand-computer assemblage produced new mathematics. One finds a similar materialist reading of inventive diagrams in the work of the philosopher of mathematics Gilles Châtelet (2000), who argues that a diagram is an experiment at the threshold between the actual and the virtual. Châtelet (2000) discusses the mathematical diagrams of Oresme, Cauchy, and Grassmann as material interventions, cutting up space, folding surfaces, and multiplying dimensions. De Freitas and Sinclair (2012, 2013, 2014, 2015) have used Châtelet's ideas to study the diagram-gesture nexus in mathematics classrooms, looking for how the "new" comes into being through these kinds of material encounters.

In this paper, I draw on the philosophical insights of Michel Serres (2011) and Brian Massumi (2011) to explore the materiality of perception in mathematics education. Author of over 50 books, Serres is one of France's most prominent philosophers and has written extensively about mathematics and science, while Massumi's work, also extensive, focuses on perception, sensation and affect. ${ }^{1}$ Theories of perception are central to embodied mathematics and play a pivotal role in both cognitive and neurological approaches to mathematics education research. This paper looks to philosophy for ways of understanding the speculative nature of perception, with the belief that philosophy offers important insights into empirical practices. Rather than study perception as the cognitive integration of multiple distinct sensory systems, my aim is to direct attention to how perception is a highly provisional material encountering between bodies. Rather than center the rational humanist subject as the synthesizer of sensory data, performing acts of discernment and judgment that collect and correlate disparate information, I explore how perception is dispersed across a material field of sensation. Developments in physiology have altered our understanding of how the eye works, pointing to how vision is a highly speculative act that entails a fusion of all sensation-vision is inherently haptic, sonic, and olfactory (Massumi, 2011). In other words, students never just register visual information-they "see" or rather absorb a (con)fusion of sensations that cannot be said to be possessed or processed by a single sense organ. The consequences of such a theoretical shift are significant in rethinking what a body can do, perhaps through technology and various material media events (Hinderliter et al., 2009). The implications of this for how we work with dis/ability in

\footnotetext{
${ }^{1}$ See for details http://senselab.ca/wp2/. I also draw from Gilles Deleuze $(1993,1994)$ whose work strongly influenced both Massumi and Serres.
} 
mathematics classrooms are substantial because it foregrounds and problematizes the relationship between sense (as meaning) and sense (as sensation). It also allows us to study the way that bodies are provisionally and temporarily enabled, directing our attention to the temporal contingency of dis/ability.

The ideas discussed in this article relate to recent research in mathematics education on how mathematical knowledge is coupled in fundamental ways to the body (Alibali \& Nathan, 2012; Nemirovsky \& Ferrara, 2009; Núñez, Edwards, \& Matos, 1999). This work points to the "sensuous" ways in which students learn mathematics and the ways in which mathematical signs and signification always entail materiality (Radford, 2009). I share with Roth (2015 a, b) a conviction that research on the body in teaching and learning requires a careful philosophical inspection of the virtual dimensions of perception and that animation (rather than enactment) captures the doing of mathematics. In this paper, I take up the question "what can a body do?" as a provocation to consider the possibility of radically different sensing bodies and to explore the implications of this in relation to mathematics education. Whether these be technology-enhanced or differently organized bodies, such a question contests assumptions about pre-given sensory modalities with definitive capacities. My hope is that this kind of philosophical and speculative work opens the future to alternate ways of becoming embodied in mathematics classrooms:

Thus the question "what can a body do?" is a pressing one, since the answer is not given in the current bodily arrangement. According to Deleuze, the body is always in a process of becoming, and potentially becoming differently perceptually enabled. The future of a body is unscripted, the sense organs unfinished and open to reconfiguration and dispersal. This posthumanist approach to sensation allows the body to break free from the confines of current perceptual organization, and demands that we recognize the human body in all its potentiality, even in our current classrooms, where bodies can be seen as differently abled and differently organized rather than disabled or distracted. (de Freitas \& Sinclair, 2014, p. 145)

The case of fractal diagrams underscores how the senses are unspecialized and can become differently organized. It is only through habit that the eye becomes specialized, that is, through repeated acts of habit the eye contracts light in particular ways. Just as digital fractal graphics became incorporated into the perceptual habits of Mandelbrot, Douady, and Rössler, students in mathematics classrooms can and do become initiated into new perceptual habits of sensing and making sense. In shifting our attention to the provisionality of perceptual habits and the materiality of the media in question, we can explore how these habits might be different. Consider for instance the distinctive nature of the small, intense, and repeated hand movements found in current touchscreen apps designed for mathematics learning. Various math apps entail dragging geometric objects on the screen, zooming in and out with the pinch gesture, and also changing the value of numerical parameter with a flicking gesture. The small, quick, flick gesture produces a new number and thus alters the very concepts of number and measure that were tied to previous forms of embodied action. The combined effect of gesture and touch is clearly related to the particular media in question.

All of the mathematicians discussed above emphasize the corporeality and material physicality of mathematics as a creative activity and a media event. The term media event refers to the often overlooked material media involved at such moments, including paper, 
dot-matrix printers, blackboards, and multi-touch ipads. I follow Vogl (2007) and others in materialist media studies in using the term media event to emphasize the event-nature of these material encounters:

These are events in a particular, double sense: the events are communicated through media, but the very act of communication simultaneously communicates the specific event-character of media themselves. Media make things readable, audible, visible, perceptible, but in doing so they also have a tendency to erase themselves and their constitutive sensory function, making themselves imperceptible and 'anesthetic'. (Vogl, 2007, p. 16)

Media events play a significant role in redefining what sensory perception entailsindeed perception is a media event whereby the senses are created anew (Vogl, 2007). Building on my previous work on the mathematical event (de Freitas, 2012, 2013) and my work with Nathalie Sinclair on inclusive materialism and dis/ability (2014, in press), this paper delves further into some of the pertinent philosophical literature informing this work.

\section{What can a body do?}

All bodies are imbricated within various interlocking material systems; a body is individuated as a body through a process of contraction and expansion that sustains a particular "centre of indiscernibility."(Bergson, 1988/1896) The encounter between bodies is always dynamic and shifting since "no one occupies a completely stable, immobile perspective from moment to moment of perception." (Rush, 2008, p. 3) To "perceive" is actually to engage with this relational environment in such a way as to fold back into it. This approach to perception underscores how our relations are sustained through continuous modulation (instead of Cartesian subject-object binaries). It also highlights two relational concepts: proprioception (the sense of being in or out of balance) and parallax (the effect of perspective on the apparent positioning of two objects). Parallax and proprioception are perceptual processes by which relations within the material world are modulated, and they highlight the relational aspects of perception. As one moves, the "proprioceptive potentialities" (p. 38) of the body are continuously reconfigured, as are the relative locations of objects in the foreground and background. Coole (2010) suggests that corporeal space is "lived spatiality, oriented to a situation wherein the lived/living/lively body embarks on an architectural dance that actively spatializes (and temporalizes) through its movements, activities, and gestures." (p.102). A related concept in body studies is kinesthesia. Sheets-Johnstone (2011) argues "not only is our perception of the world everywhere and always animated, but our movement is everywhere and always kinesthetically informed" (p. 113). Kinesthesia refers to the ability of the human body to feel its own movement and states and thereby contributes to the sense that "oneself" is the source of such action (Streeck, 2013). Rather than essentialize this sense of willfulness or intentionality or attribute it to intuition, I want to explore how the proprioceptive potentialities of the body are provisional and indeterminate.

It is through the elastic potentiality of the body, its responsive stretching in relation to other bodies, that the human body begins to habituate to particular kinds of actions. In this regard, the philosopher of science and mathematics Michel Serres (2011) suggests that there is a "material mimicry" that needs to be better studied in teaching and learning in mathematics. 
This is not to undermine the importance of conceptual understanding, but to begin to grasp the tiny muscular actions that are entailed in every kind of learning encounter (the tapping on a touchscreen, for instance). It is not that teaching is exhausted through this physical mimicry, but that learning entails the body in ways that we are yet to understand. Serres (2011) describes this aspect of learning as unconscious and sometimes trance-like:

The teaching body dances its knowledge softly so that the audience will, like it, go into a trance and so that, through virtual mimicry of its gestures, a few ideas will enter their heads via the muscles and bones, which though seated and immobile are solicited, pulled toward the beginnings of movement, perhaps even by the written work's little jig. (Serres, 2011, p. 96)

Again, this is not to dismiss the importance of understanding as we have come to conceive of it in mathematics education, but to study the way that learning also entails a material mimicry "without understanding" in that one engages with rather than recognizes that which one learns. Serres is not advocating for rote memorization, but for an appreciation of how learning occurs, in part, without our conscious awareness. Thus, there is "nothing in knowledge which has not been first in the entire body, whose gestural metamorphoses, mobile postures, very evolution imitate all that surrounds it."(p. 70) This is a body that is enmeshed or mixed with other bodies, continuously altering the assemblages it forms with other bodies. And it is precisely because of this ongoing change in the mix that there is a potentiality in the body that outstretches current epistemologies and demands that we rethink the contours of the body:

How do we define a body given over to so many poses and signs: when and under which form is it itself? How do we get beyond so many differences according to the person: when and under which form is it us? These multiple postures prevent us from saying. My body and our species don't exist so much in concrete reality as 'in potency' or virtuality. (Serres, 2011, p. 52)

And yet if the body is more potential than actual, more a process of becoming than being, how does such a body come to know mathematical concepts that seem so fixed and timeless? Serres (2011) argues that mathematical concepts, more so than other kinds of concepts, seem to capture the body's fluidity and "indefinite capacity to transform" its material relations (p.94). Mathematical events of creation, he argues, depend on "infinitesimal intuitions" or "petites perceptions" when consciousness submits to the impersonal pre-individual mobility of material entanglements. As a philosopher of mathematics, he unpacks mathematical invention for how the material configuration in that moment of invention - body, culture, technology — demands a new way of sensing and making sense. Of course, mathematical inventions do not occur in one moment, but over long periods of time and as a result of sustained activity by a community of practice. But this does not curtail studying the material habits and the material circumstances of those people involved for how these habits and circumstances factored into the emergence of new mathematics. We do this regularly in claiming that mathematics has utility when we argue that particular ancient number systems were based in particular mercantile practices. In fact, the utility of mathematicshow it applies so effectively to the physical world - may have distracted us from other ways in which the mathematical and the material are coupled. Why not attend at a more molecular level to the habits of the human body as it partakes in these practices? Not so much for justifying (school) mathematics because of its utility, but perhaps for the sake of better understanding our convictions about that utility. One of the perrenial questions in the philosophy of mathematics pertains to its 
utility and uncanny application to the material world. When we consider the radical potentiality of a body, how its capacity to affect is objectively unknowable (because that capacity is entirely relational and emergent), we are forced to rethink concepts like utility. This shifts our attention to explanations of invention and descriptions of material relationships that condition the emergence of the new. Serres (2011) describes the event of Leibniz trapped on a boat for days, at the mouth of the Thames, detained by contrary winds, his body "thrown into a thousand shifting inclinations." Pouring over his notes as the boat rolled, he formulated the details of the infinitesimal calculus. Or did he? Such an experience at sea was not, of course, a causal condition for the invention of the calculus, and it would be foolish to suggest so. The anecdote, however, like all stories that relate bodily events to the creation of enduring ideas, is compelling because of the way it undermines the necessity of invention. By attending to the body at such moments and its changing relationship with other moving bodies, we come face to face with the contingency of mathematics. Serres discusses other mathematical inventions, grounding each in the material entanglement of bodies:

Do you want to invent mathematics? Consult your body, the devil take Plato; the sublime philosopher claimed that the ignorant slave, as staged in the Meno, had forgotten that he knew geometry, while the theory of the Forms hid from its author and two thousand years of servile mimicry this glaring truth: all bodies know geometry and each is ignorant of it. Blind to the body's riches, we don't even see what those [who] do see them are doing: creators owe their discoveries to an exquisite proprioceptivity. (Serres, 2011, p. 146)

We hear this "exquisite proprioceptivity" in the comments of Mandelbrot, Douady, and Rössler above. When Mandelbrot speaks of the unspecialized eye, he is underlining the adaptability of the eye and how vision becomes vision as a response to the environment; when Rössler speaks of his "ear pop" and his "nose perception," he directs our attention to the ways that his whole body was engaged in the diagramming experiment. As researchers in mathematics education, this kind of attention to the body demands that we study the minute sensations that contribute to our students' learning and invention of mathematical concepts. In attending to the potentiality of the body, one begins to imagine how the body could be differently configured. Instead of speaking about the affordances of any particular sense (vision, hearing, touching), one can study the way that sensation operates chaotically below the level of perception. Here, I draw on the philosophy of Deleuze $(1981,1993,1994)$ who directs our attention to the same "infinitesimal intuitions" or "petites perceptions" that operate at microscales beyond the limits of perception. Rather than treat perception as the synthesis of distinct micro-perceptions, he suggests that these infinitesimal intuitions traverse and indeed sustain a field of sensation that continuously binds differently modulated bodies together. Thus, perception is an act of contraction or expansion of the various flows of sensation across this field. Perception occurs as a media event occurring across the material fabric that binds all bodies in a collective. This continuous recalibration or modulation operates beneath the apparent judgments entailed in perception. Indeed, perception is less about synthesis of distinct sensations and more about an investment in a collective resonance of intensity - a resonance effected across a collective, rather than by an individual — so as to form folds in the tissue of shared experience. This approach treats learning as a rhythmic folding of sensations, a modulating intensity that traverses the tactile surface of our material entanglements. In a similar fashion, Roth (2015 b) speaks of the "kinetic melodies" that might describe the various eddies and flows of thought circulating and sustaining (and exceeding) the activity of mathematical graphing. This approach applies to entanglements between bodies, concepts, and signs. As Smith (2003) puts it, "Beneath concepts, one always finds rhythmic blocks of 
complexes of space-time." (p. xix) Rhythm is likewise the pre-perception of a diagram: "it is rhythm itself that would become the Figure, that would constitute the Figure." (Deleuze, 1981, p.60) If sensation is a modulating wave of intensity, then the individual human body is a media event of resonance and convergence, with rough fractal borders. This is a body with potentially different perceptual capabilities or different calibrations of sensation, depending on the encounters. Thus, the entire concept of perception must be studied less as a process of receiving and synthesizing information and more as a relational contraction of energy and affect. In the next two sections, I discuss theories of perception that develop this approach and I show how these theories shed light on the role of diagrams in mathematics.

\section{The virtuality of perception}

No matter what one's theory of perception, it seems indisputable that the practice of mathematics over the last few millennia has in large part involved acts of graphism or inscription. Whether one be scratching away on slate, parchment, paper, or screen, mathematical activity often entails a material-semiotic activity that engages various kinds of two-dimensional surfaces. Such a claim is not meant to dismiss the role of other sensory modalities in doing mathematics (indeed, one of my aims is to show how these modalities are always entangled), but to draw attention to the pivotal role of one practice in particular - that being the gestural act of marking up surfaces. If we look more closely at these graphing habits as a form of sensing, we see that the surface itself-the unique ways in which the surface offers up a material ground - plays a significant role in structuring the way we make sense of our sensation. When we draw a perspectival image of a cube and conjure depth using a dotted line or when we create a small semi-circle around a singularity to indicate how we will integrate over the complex plane, we are simultaneously construing the limits of the sensible while engendering another (presently) untouchable world beyond the surface. If the surface is a locus of sense in that "signs" would be without sense if they failed to engage with the surface organization, then we need to study the way encounters with surfaces in our mathematics classrooms determine the limits of the sensible. Perhaps Belting (2005) helps us understand these habits by suggesting that we should attend more carefully to the melding of image to surface. In his evocative words, "images live, as we are led to believe, in their media much as we live in our bodies." (p.306) He puts forward a historical and anthropological study of the image, arguing that humans have always invested their images with flesh and dimensionality so that they might engage with them by way of the surface. We animate the media so that our images will come alive. In other words: "Animation, as an activity, describes the use of images better than does perception.” (p. 307)

The philosopher Brian Massumi (2011) argues against the language of "cross-modal transfer" used in perception studies because it does not address the way that the senses are lived in. The concept of "transfer" imposes a model on sensing that treats objects of perception as given in some form of stasis and fails to account for the sensing of relationality and mobility. Instead, Massumi describes perception in terms of "cross-modal fusions" in that touching, kinesthesia, smelling, and other kinds of sensing "inhabit the event of vision" (p. 74) or any other occurrence of sense. Rather than talking about transfer between sensory modes, he suggests that the senses are always already fused - not correlated, nor merely coupled, but literally fused together. They take each other up, never functioning alone. As Massumi suggests, "The senses only ever function together, fusionally, in differential contrast and 
coming-together."(p. 75) The radical implication of such work is that a purely fusional sensory system - if examined at the micro level-points to the occurrence of immanent and amodal sensing. In other words, a great deal of sensing is outside of modality. It is neither this mode nor that one. It is a lived-in sensing that is pre-perception and pre-apprehension (insofar as these are conceptualized in perception studies or semiotics).

What this means for the study of sense and sensing in mathematics classrooms is that we need to reckon with the virtuality of perception. Students never just register visual information from that which is in front of their eyes - they see potentiality, relationality, mobility, occurrence, and the future. Students are not seeing an object, they are seeing an event: "An object's appearance is an event, full of all sorts of virtual movement."(p. 43) In other words, we live on "speculative investments,"(p. 88) as though we were surfing "the front edge of a wave-crest." (p. 69) Perceiving an object entails a prehensing of our body's potentiality to walk around the object, to reach out and touch the object, to visualize the object, to weigh it, to smell it, to envision its future. And yet it is hard to imagine how such bodily investment is fully at stake when mathematics students engage with a mathematical diagram in a conventional classroom. One needs to look more closely at the specific kinds of diagrams and media events entailed in such classrooms.

Mathematical diagrams are different from photographs and paintings in that they do not position a viewer in one designated or legitimate location from which to observe since they break with the rules of typical perspectival perception (Bender \& Marrinan, 2010; Lechte, 2011; Millar, 2006). For instance, the use of shadow, depth, transparency, context, and white space is operative in ways that allow for diverse entry points into the diagram (de Freitas, 2012). In not positioning the viewer in one particular location to view the image, mathematical diagrams invite a more active and yet virtual engagement than other images. As Bender and Marrinan suggest in their cross-cultural study of diagrams, "users of diagrams, unlike viewers, are functional components inseparable from the system in which they are imbricated. They are empowered to initiate a process of correlation even as they realize their subjectivity presence is liminal-almost non-existent." (Bender \& Marrinan, 2010, p. 72) In other words, users of diagrams are not hailed or addressed as embodied observers located in one position, which is, for the most part, the way photographs and paintings function. This "liminal" location can cause confusion for some students, since the body is hailed less as a coherent individuated subject and more as a potential movement. But if students are invited to partake in - or become aware of and indulge - the inherent mobility of their embodiment, they can begin to comfortably listen to the diagram (the "ear pop") and become part of that mobile field of sensation. If students surrender to the apparent indifference of the diagram to their observation, that is to say, the way in which the diagram refuses to position and fix a viewer, they can begin to leverage modes of engagement that differ from those associated with other images like photographs and paintings. This suggests that students who are successful at making sense of diagrams may engage with this liminal positioning. Some students may turn away from such a demand, while others accept their ambivalent status, engaging with the diagram through acts of speculation. In the section below, I discuss the specific material conditions of blackboards for how they invite speculative investment in mathematical diagrams.

\section{Blackboards and media events}

The blackboard is iconic of mathematics and remains the preferred surface (rather than the whiteboard or the smartboard) for many mathematicians. Greiffenhagan (2014) cites a number 
of mathematicians who explain this preference in terms of the way the materiality of the board allows the mathematics to come alive: "Blackboards are best for maths (in my opinion) because $[. .$.$] the need to see ideas materializing in front of you." Another stated that by using$ blackboards, "mathematics becomes visible as a process, not just as a product. This is particularly important in teaching." Yet another compares this experience to PowerPoint lectures:

In math, we are not just teaching facts, but a way of doing things. Presenting this as a sequence of (well-prepared) computer slides will not give them the right impression of how mathematics develops, and thus miss one of the crucial points of why we still have lectures." (p. 522)

Instead of simply dismissing these remarks as evidence that habits are hard to change, it seems worth attending to the specific material aspects of chalk and blackboard that contribute to sensory habits in teaching and doing mathematics. How do blackboards, more so than white boards, allow for particular kinds of sensory habits around diagramming? Might the specific haptic, visual, and sonic aspects of the encounter-for instance, the speed with which the hand is able to move with the chalk, the fuzziness of any white line that is drawn, the sound and heat that are produced-be bound or fused with the kind of mathematics that is practiced? Might the sense-making and the sensation be intimately linked for these mathematicians?

Massumi (2011) discusses the work of the renowned American philosopher Charles Peirce, whose theory of signs has been taken up and used extensively in mathematics education research. Massumi points to Peirce's interest in the materiality of media and his analysis of what is entailed when we create a mathematical diagram on a blackboard. Peirce shifts our attention away from the signification of the drawn line and toward the surface perturbation that is effected by the chalk. He claims that this appearing of the white line is quite literally the "springing up of something new" in the material plane (p.89). As Peirce suggests in the quote below, the line is actually a surface - an "oval line"-because of the way it bleeds into the material of the blackboard. In other words, the drawn line is always a material line, occupying the continuous black space of the board, while the boundary between the white and the black is a virtual relation, in not belonging to either the white or the black. Thus, there is a discontinuity affected through the drawing of the chalk line in a mathematical diagram, but it is virtual. The continuity of the line, says Peirce, is merely a reflection of the continuity of the board on which it is drawn, whereas the discontinuity rendered by the line (the separation of two regions) is purely virtual and indeterminate, sustained through a relational "pairedness" that inheres in the event of diagramming.

Let the clean blackboard be a sort of Diagram of the original vague potentiality, or at any rate of some early stage of its determination .... This blackboard is a continuum of two dimensions, while that which it stands for is a continuum of some indefinite multitude of dimensions .... I draw a chalk line on the board. This discontinuity is one of those brute acts by which alone the original vagueness could have made a step toward definiteness. There is a certain element of continuity in this line. Where did the continuity come from? It is nothing but the original continuity of the black board which makes everything upon it continuous. What I have really drawn there is an oval line. For this white chalk-mark is not a line, it is a plane figure in Euclid's 
sense - a surface, and the only line that is there is the line which forms the limit between the black surface and the white surface. This discontinuity can only be produced upon that blackboard by the reaction between two continuous surfaces into which it is separated, the white surface and the black surface. The white is a firstness - a springing up of something new. But the boundary between the black and white is neither black, nor white, nor neither, nor both. It is the pairedness of the two. It is for the white the active secondness of the black; for black the active Secondness of the white. (Peirce, 1992, p. 261-262, cited in Massumi, 2011, p. 89)

Peirce is underscoring the materiality of graphism and pointing out how the geometric line is a molecular smudging that links the continuity of matter with the continuity of the number line. Extension and measure are coupled through this media event. At the micro level, the molecular bonding between chalk and blackboard is never definitive or static. If we were able to perceive at this level, we would grasp the incredible mobility at play in the diagram on the board. There is no static figure or stillness of an image - the line is all movement and perturbation with the material surface. The materiality of graphism exceeds the determination of foreground and background, inside and outside, and other framings of sense. As Massumi (2011) suggests, the line does not enclose or divide regions on the board - it actually activates or animates the board. By examining the act of mathematical diagramming on blackboards at this microscale, we begin to understand in new ways why so many mathematicians have been drawn to this medium. On the blackboard, the line is a virtual line, a line that sustains the gestural mobility of its being drawn. The blackboard line taps into the materiality of the surface and actualizes a perceptible difference. How is this different on a whiteboard or with multitouch iPads? Massumi helps us understand the process of co-surfacing of the line and the plane. This is an act that does not detach or separate but seems to involve some sort of mutual entailment. If we keep drawing and multiplying the lines, proliferating the marks, and continuing to repeat our encounter, we see that a figure becomes visible. Massumi offers the following diagram of an oval composed of straight lines (Fig. 3).

While Peirce attended to the line as a chubby oval line, this series of straight lines is now perceived as an oval. With repeated scratches, an oval edge emerges with visible thickness, creating another surface effect of inside and outside. We perceive a still figure or boundary curve, despite the fact that the gestures that marked the page were furtive straight scratches. The "first furtive gestural line" that evoked and provoked the potentiality of the surface has given way to the stability of the oval curve. The motility of the line (and the fact that our hand and body were engaged in its making) is eclipsed by the stillness of the figure and, with persistence, becomes a mathematical form. In mathematics, we are inclined to move rather quickly to the static form, forgetting the gestures that animated it. In other words, we move from the "emergent proto-figural activity" to the "resulting figurative annulment" of that bodily engagement with the surface (Massumi, 2011, p. 92). Although our unconscious eyes may move back and forth between the two, as Massumi (2011) describes:

Looking more or less closely, focusing more or less attentively, the eye oscillates between the annulment of the process and its activity. Flicker. Between fully-hatched stability and continuing, cracked emergence. Flicker. Between the made and the making. Flicker. Between seeing the figurative stability and seeing the imperceptible float of figural potential. Flicker. The eye tires of the flicker. It habituates to bridge-level stability. The eye is the organ of habitual oversight. The figure is an habitual inattention to the imperceptible in vision. (Massumi, 2011, p. 93) 
Fig 3 (Massumi, 2011, p. 91)

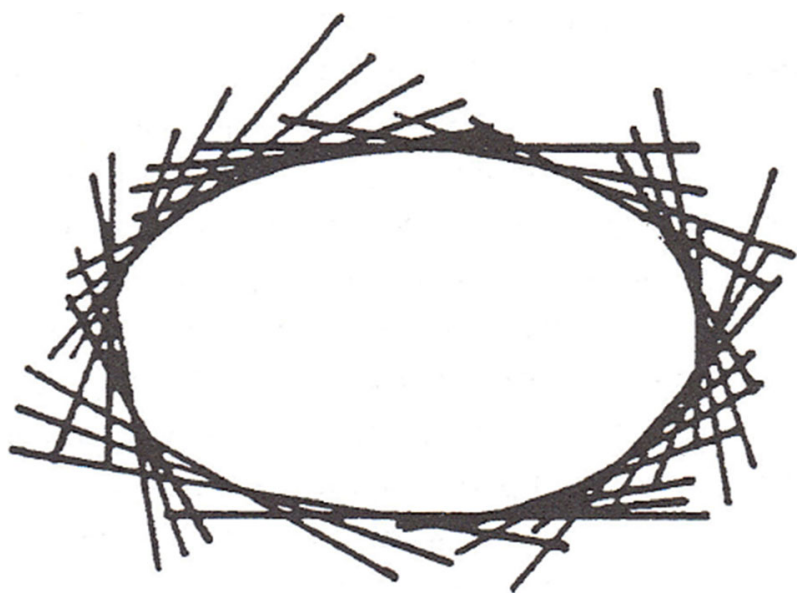

Let us repeat this incredible refrain that Massumi offers us: "The figure is an habitual inattention to the imperceptible in vision." In other words, the diagram or figure is the result of a habitual oversight, an entrained blocking of the potentiality or virtuality ("the imperceptible") that is always entailed in perception. Roth (2015 a) explores a similar approach using the concepts of "invisible immanence" and "pure multiplicity" to describe how a student manipulates tangrams and suddenly "sees" a new shape arrangement. Through this analysis of sense and sensing, we see how vision is inhabited by touch, smell, taste, and proprioception, etc. and that we perceive the diagram (or the tangram) through tendencies of inattention. Beneath perception is an "undifferentiated sensory experience." (Massumi, 2011, p. 95) Perception of diagrams is thus a complex process of fixing the sensation and stopping the mobility that inheres in the field of sensation. Students' perception encounters the blackboard itself, then also the mathematical markings on it, then the figure and finally the form. The process might be described in the following sequence: "blank slate - singular proto-figure - stable figure-object identity - ideal standard-general model." (Massumi, 2011, p.100) In arriving at the general model, we commonly erase all memory of the materiality of the surface with which we engaged, forgetting the process or event-nature of perception.

Two important questions emerge from this analysis: first, how does sense-making in classrooms - where students are surrounded by screens, boards, and paper-depend on a speculative investment in the stillness of the figure so that the student might join fully in the community of mathematical meaning making? Second, what is lost when we train our students' eyes to see only the still figure and forget the turbulent potentiality of the material surface? Might there be a good reason to explore more dynamic surfaces that allow for figures to sustain that potentiality? How do dynamic digital screens factor into this way of thinking about perception? In the context of mathematics education, where the figure is more often taken as a copy of an ideal form, the suggestion that it might be "habitual inattention" invites us to revise theories of multi-modal integration. If the diagram is, as Massumi suggests, the "fill" and the "speculative investment" and if perception involves a "chaos of vision" (eyes jittering, light scattering) and we invest in the diagram via other sensory encounters, 
then we need to reckon with the ways in which our students might not have had opportunity to habituate into this kind of investment. Moreover, the question arises as to how our technologies are changing this aspect of learning. Consider, for instance, the way that touchscreen technologies (such as interactive whiteboards, iPads, tablets, and related software) offer up a new kind of surface for engagement, reconfiguring our sense of touch-and what is entailed in it - through the actions of tapping, sweeping, and finger-release. The touchscreen reconfigures our sensations, bringing together haptic (touch) and visual perception in new ways and altering what we take to be normal mathematical practice (Sinclair \& de Freitas, 2015). Material engagements with surfaces more generally, be they touchscreen or more traditional blackboards, partake in the making of mathematics. Surfaces are here considered broadly to be sensory boundaries - in other words, there can be sonic non-visible surfaces. It is through these encounters with diverse surfaces that students and teachers collectively reconfigure the relationship between mathematics and the sensible, where the sensible refers to what makes sense and what can be sensed. In the next section, I discuss implications for how we approach mathematics dis/ability.

\section{Political implications for classrooms}

Everyday, material practices are central to experiences of mathematics educationmaterial practices of sense and sensation configure what is taken to be visible, touchable, and sayable, while also delineating the contours of embodied subjectivity. In other words, sensing and making-sense in mathematics education are political practices precisely because they are the "common" practices by which "the distribution of the sensible" is achieved (Rancière, 2004). For this reason, focus on the potentiality of the body has socio-political consequences for how we conceptualize dis/ability in mathematics classrooms. The human body becomes differently abled when we consider how contemporary assemblages of human and non-human engender new kinds of experiences.

Drawing, writing, and inscribing of "abstract" signs on various surfaces is both a perceptual act and a political act, insofar as this kind of mathematical "doing" determines (and does not just reflect) the limits of the sensible for our communities of practice. Thus, sense and sensing correlate to the mapping of particular "perceptual coordinates" in mathematics classrooms, and regimes of perception in mathematics education actually produce the limits of what is sensible and what is thereby endowed with a "common language" for those who are within that elite community of practice.

The ways in which mathematics is represented, communicated, and explained tacitly privilege certain sensory capacities. While there are many kinds of learning barriers (for example, deaf and blind learners are less likely to be taught by mathematically trained teachers), there is a growing awareness of the way in which certain sensory modalities are privileged, often only implicitly, in school mathematics (Healy \& Fernandes, 2011; Nunes, 2004). De Freitas and Sinclair (2014) note that

In seeking to understand how deaf and blind learners develop mathematical expertise, contemporary researchers have made evident two important issues: (1) the loss of one sense may change the way other senses are used, which may lead to certain opportunities 
that often go untapped ${ }^{2}$; and (2) mathematics itself changes under different sensory organisations. (p.148)

For instance, while deaf children often underperform on counting tasks, their more spatial way of thinking about numbers allows them to perform better than hearing learners on tasks such as counting backwards and "What number comes after this one?" (Nunes, 2004). The mathematics curriculum is thus formatted by certain assumptions about which sensory skills should be favored. The curricular emphasis on alphanumeric aspects of mathematics, for instance, works against students with exceptional spatial skills. People diagnosed with dyslexia may struggle with procedural learning and rote memory tasks, but their memory of phenomenological details - details pertaining to physical aspects of an experience, such as tactile, motor, or spatial arrangements - exceeds that of non-dyslexics (Eide \& Eide, 2011).

Manning (2007) suggests that a politics of sense must rethink the senses as "expressions of moving bodies," (p. xiii) bodies that are no longer stably identified in one particular space-time location and defined by borders. Such an approach to the body entails an equally destabilizing approach to space and time: "The body does not move into space and time, it creates space and time: there is no space and time before movement."(p. xiii) If sensing is constitutive of space and time in this ontologically radical way, then our bodies are engaged in micropolitical acts as they "sense" the world. Rather than ask what a body is, Manning and others now ask, what can a body do? Clearly, this is a question that sees the body as a site of political action. Movement becomes the defining quality of political life in that it determines the space-time coordinates of the body, and its absence all too often indicates the imprisonment or disempowerment of the body. For Deleuze and Guattari (1994), movement is an intrinsic quality of becoming, and possibly a condition of it. Power is incarnated in a moving body that deviates from the laws that govern its location. A thing becomes a body when it "undergoes changes in coordinates" (122), when it ruptures a space and when it captures (composes) an invariant under a group of transformations. Manning (2007) makes this more explicit, with reference to touch: "Sensing bodies in movement are ontogenetic. They are ontogenetic because they are always in genesis, in a state of potential becoming." (p. xxi)

One might claim that this is a weak politics that cannot address the reality of sociocultural inequity but perhaps this kind of attention to sense, sensing, and making-sense is in fact the best way to study the political configurations of perception in mathematics classrooms since capital relies on an esthetic regime of appearance in order to compartmentalize bodies. Moreover, shifting the political toward the examination of practices of sense and sensing allows us to show how the body is always in excess of these regimes of signification, how sensing is ultimately without measure. As Manning submits:

If we understand sensing as more than a motor response, we are in a position to explore the uknowability of sense. In other words, sensing need not express a sensation we have already experienced. To sense may also be to know differently, in excess of my current appreciation of 'my' body. (p. 131)

By examining visual perception as something open to philosophical reflection, we can begin to track the way reconfigurations of the sensible-where the sensible refers to what makes sense and what can be sensed-are forged into new political relations within

\footnotetext{
2 This is not an "accommodation" approach, whereby one sense takes up the absence of the other. Rather, we emphasize in this claim that the sense organs are plastic, or open to mutation, even within the lifetime of the individual.
} 
educational contexts. In order to be more inclusive of other ways of sensing in our classrooms, we must decenter our own regimes of sensing (Panagia, 2009). The pluralization of values in a community depends on a political aesthetics whereby norms of sensation are shown to be contingent and open to contestation. Thus, research on bodies in mathematics classrooms has radical implications for how we research the politics of mathematics education. As Panagia (2009) explains, the political often plays out at the level of sensation since the source of the political often entails "a dissensus of sensation that disrupts our confidence in the correspondence between perception and signification."(Panagia, 2009, p. 5) The term "dissensus" captures the way that dis/ability is often simply a different way of sensing. Focus on perception and sensation is one way of digging into the politics of mathematics achievement and thereby links to cultural studies of mathematics education at the more macro level. One grasps the incredible truth of Panagia's claim that "the first political act is also an aesthetic one" (p.9) when studying how mathematics education operates through regimes of perception and affinities of sensibility that cohere the community of practice around particular habits, pathologizing students as disabled or deficit if they perceive differently.

This approach has been taken up in the critical dis/ability literature. Shakespeare and Watson (2001) argue for an affirmative movement, where dis/ability is redefined as "[t]hat in the body which exceeds deterministic efforts to predict a life trajectory" (Snyder \& Mitchell, 2001, p. 377). And the disability theorist Goodley (2009) suggests that "the metaphor of the body as organism refers to an endemic societal view of the body as sovereign self: contained, knowable, measurable, and dis/abled." (p. 264) These developments in dis/ability theory are now studying sensation as that which is dispersed across an event, where a body is always becoming individuated with a provisional set of organs open to constant reorganization.

\section{New directions for research}

In this paper, I have focused on the potentiality of the body, exploring how perception is a provisional media event, and how mathematics teaching and learning involves such media events. Analyzing visual perception for how it operates through virtuality and speculative investment allows us to imagine an unscripted future body with capacities unrecognizable to us today. One might contend that the sensory capacities of the hominin have been basically the same for 5 million years and that any prosthetic extensions are extraneous to an essentialized human form. I would argue that such an approach to the body fails to reckon with the radical material differences entailed in cultural transformations. I do not deny that bodies of all kinds have orientations and particular "I cans" that are related to the particular configuration of bodies in relation. As Sheets-Johnstone suggests, "creaturely forms [have] certain potentialities of movement and not others in virtue of the bodies they are, and in turn [have] certain conceptual potentialities and not others." (Sheets-Johnstone, 2012, p. 20) But as Mandelbrot declared as he ventured into new digital media, "the eye is unspecialized."

The body's potentialities are not exhaustively determined by the current bodily arrangement, and new kinds of material encounters will entail new bodily arrangements. My argument pushes beyond typical phenomenological approaches to the body that emphasize that the body is the "zero-point" from which the world unfolds (Ahmed, 2010). I have argued in this paper that we can and should destabilize this zero-point or starting point to see that one's positionality is provisional or relational. The current orientation of the body - and its configuration of sense organs - could be entirely different in the future, so different that the very idea 
of a "zero-point" might be completely revisioned. As Serres (2011) argues, mathematics itself seems to offer strong evidence for this claim. The mathematicians Lucian Boi, Benoit Mandelbrot, Adrien Douady, and Otto Rössler all partook in a radical reconfiguring of mathematics and simultaneously a reconfiguring of their own sensory engagement with mathematical concepts. Reconsidering perception as a speculative investment has important implications for mathematics classrooms because it points to how the "distribution of the sensible" formats curriculum. The regimes of sensation that are validated in our classrooms through our engagement with particular surfaces (whiteboards, elmo projectors, iPads) must be examined for how they structure student experiences. Micro-sensory practices associated with particular mathematical practices can show us how dis/ability is produced therein. This opens up all sorts of new directions for research in mathematics education so that we might better understand how the body factors into student success.

Open Access This article is distributed under the terms of the Creative Commons Attribution 4.0 International License (http://creativecommons.org/licenses/by/4.0/), which permits unrestricted use, distribution, and reproduction in any medium, provided you give appropriate credit to the original author(s) and the source, provide a link to the Creative Commons license, and indicate if changes were made.

\section{References}

Ahmed, S. (2010). Orientations matter. In D. Coole \& S. Frost (Eds.), New materialisms: Ontology, agency, and politics (pp. 234-257). London: Duke University Press.

Alibali, M. W., \& Nathan, M. J. (2012). Embodiment in mathematics teaching and learning: Evidence from learners' and teachers' gestures. Journal of the Learning Sciences. (Special Issue on Embodiment in Mathematics), 21(2), 247-286.

Belting, H. (2005). Image, medium, body. Critical Inquiry, 32, 302-319.

Bender, J., \& Marrinan, M. (2010). The culture of diagram. Stanford: Stanford University Press.

Bergson, H. (1988/1896). Matter and memory (N.M. Paul \& W.S. Palmer, Eds.). New York: Zone Books.

Châtelet, G. (2000/1993). Les enjeux du mobile. Paris: Seuil. In R. Shore \& M. Zagha (Eds.), Figuring space: Philosophy, mathematics and physics. Dordrecht: Kluwer

Coole, D. (2010). The inertia of matter and the generativity of flesh. In D. Coole \& S. Frost (Eds.), New materialisms: Ontology, agency and politics (pp. 92-115). London: Duke University Press.

de Freitas, E. (2012). The diagram as story: Unfolding the event-nature of the mathematical diagram. For the Learning of Mathematics, 32(2), 27-33.

de Freitas, E. (2013). The mathematical event: Mapping the axiomatic and the problematic in school mathematics. Studies in Philosophy and Education, 32, 581-599.

de Freitas, E., \& Sinclair, N. (2012). Diagram, gesture, agency: Theorizing embodiment in the mathematics classroom. Educational Studies in Mathematics, 80(1-2), 133-152.

de Freitas, E., \& Sinclair, N. (2013). New materialist ontologies in mathematics education: The body in/of mathematics. Educational Studies in Mathematics, 83(3), 453-470.

de Freitas, E., \& Sinclair, N. (2014). Mathematics and the body: Material entanglements in the classroom. Cambridge: Cambridge University Press.

de Freitas, E. and Sinclair, N. (2015). The cognitive labour of mathematics dis/ability: Neurocognitive approaches to number sense. Research in Mathematics Education.

Deleuze, G. (1993/1988). The fold: Leibniz and the baroque (T. Conley, Ed.). New York: Continuum.

Deleuze, G. (1994/1968) Difference and repetition (P. Patton, Ed.). New York: Columbia University Press.

Deleuze, G. (2003/1981). Francis Bacon: logic of sensation (D. Smith, Ed.). Minnesota: University of Minnesota Press.

Deleuze, G., \& Guattari, F. (1994). In H. Tomlinson \& G. Burchell (Eds.), What is philosophy? New York: Columbia University Press.

Douady, A. (2005). Interview with Nina Samuel. In Nina Samuel (2012). The islands of Benoit Mandelbrot: fractals, chaos and the materiality of thinking. Bard graduate Center: Yale University Press.

Eide, B. L., \& Eide, F. F. (2011). The dyslexic advantage: Unlocking the hidden potential of the dyslexic brain. New York: Penguin.

Goodley, D. (2009). Bringing the psyche back into disability studies: The case of the body with/out organs. Journal of Literary \& Cultural Disability Studies, 3(3), 257-272. 
Greiffenhagan, C. (2014). The materiality of mathematics. British Journal of Sociology, 65(3), 502-528.

Healy, L., \& Fernandes, S. H. A. A. (2011). The role of gestures in the mathematical practices of those who do not see with their eyes. Educational Studies in Mathematics, 77, 157-174.

Hinderliter, B., Kaizen, W., Maimon, V., Mansoor, J., \& McCormick, S. (2009). Communities of sense: Rethinking aesthetics and politics. Durham: Duke University Press.

Lechte, J. (2011). Some fallacies and truths concerning the image in old and new media. Journal of Visual Culture, 10(3), 354-371.

Mandelbrot, B. (1982). The fractal geometry of nature. New York: Freeman.

Mandelbrot, B. (1992). Fractals and the rebirth of experimental mathematics. In H. Jürgens, H.-O. Pietgen, \& D. Saupe (Eds.), Fractals for the classroom: part one: introduction to fractals and chaos (pp. 1-16). New York: Springer.

Manning, E. (2007). The politics of touch: Sense, movement, sovereignty. University of Minnesota Press.

Massumi, B. (2011). Semblance and event: Activist philosophy and the occurrent arts. Cambridge: MIT Press.

Millar, B. (2006). The conflicted character of picture perception. The Journal of Aesthetics and Art Criticism, $64(4), 471-477$.

Nemirovsky, R., \& Ferrara, F. (2009). Mathematical imagination and embodied cognition. Educational Studies of Mathematics, 70(2), 159-174.

Nunes, T. (2004). Teaching mathematics to deaf children. London: Whurr Publications.

Núñez, R., Edwards, L., \& Matos, J. F. (1999). Embodied cognition as grounding for situatedness and context in mathematics education. Educational Studies in Mathematics, 39, 45-65.

Panagia, D. (2009). The political life of sensation. Durham: Duke University.

Peirce, C. S. (1992). Reasoning and the logic of things. Cambridge: Harvard University Press.

Radford, L. (2009). Why do gestures matter? Sensuous cognition and the palpability of mathematical meanings. Educational Studies in Mathematics, 70(3), 111-126.

Rancière, J. (2004). The politics of aesthetics: The distribution of the sensible (G. Rockhill, Ed.). New York: Continuum.

Rössler, O. (1977). Chaos is abstract kinetics: Two prototypes. Bulletin of Mathematical Biology, 39, $275-289$.

Rössler, O. (2012/2007). Interview with Nina Samuel. In N. Samuel (Ed.), The islands of Benoit Mandelbrot: fractals, chaos and the materiality of thinking. Bard graduate Center: Yale University Press.

Roth, W.-M. (2015a). The visible and the invisible: On the immanence of doing mathematics or mathematics as revelation. Educational Studies in Mathematics, 88, 221-238.

Roth, W.-M. (2015b). Excess of graphical thinking: Movement, mathematics and flow. For the Learning of Mathematics, 35(1), 2-7.

Rush, F. (2008). On architecture. New York: Routledge.

Samuel, N. (2012). The islands of Benoit Mandelbrot: Fractals, chaos and the materiality of thinking. Bard graduate Center: Yale University Press.

Serres, M. (2011). Variations on the body (R. Burks, Ed.). University of Minnesota Press.

Shakespeare, T., \& Watson, N. (2001). The social model of disability: an outdated ideology? In S. N. Barnartt \& B. M. Altman (Eds.), Exploring theories and expanding methodologies: Where we are and where we need to go (Series in social science and disability, Vol. 2, pp. 9-28). Amsterdam: JAI.

Sheets-Johnstone, M. (2011). The primacy of movement (2nd ed.). Amsterdam: John Benjamins.

Sheets-Johnstone, M. (2012). Movement and mirror neurons: A challenging and choice conversation. Phenomenology and the Cognitive Sciences, 11(3), 385-401.

Sinclair, N., \& de Freitas, E. (2015). The haptic nature of gesture: Rethinking gesture with multitouch digital technologies. Gesture.

Smith, D. W. (2003). Translator's preface. In G. Deleuze \& F. Bacon (Eds.), The logic of sensation (pp. vii-ix). New York: Continuum.

Snyder, S. L., \& Mitchell, D. T. (2001). Re-engaging the body: Disability studies and the resistance to embodiment. Public Culture, 13(3), 367-389.

Streeck, J. (2013). Interaction and the living body. The Journal of Pragmatics, 46, 69-90.

Vogl, J. (2007). Becoming-media: Galileo's telescope (B. Hanrahan, Ed. \& Trans.). Grey Room, 29, $14-25$. 